\title{
A medicalização do parto no Brasil a partir do estudo de manuais de obstetrícia
}

\author{
The medicalization of childbirth in Brazil \\ through the study of obstetrics handbooks
}

\section{Lucia Regina de Azevedo Nicida}

Doutoranda, Programa de Pósgraduação em Saúde da Criança e da Mulher, Instituto Nacional de Saúde da Mulher, da Criança e do Adolescente Fernandes Figueira/ Fiocruz.

Rio de Janeiro - RJ - Brasil

lucia.nicida@gmail.com

Recebido em 3 out. 2017.

Aprovado em 19 ago. 2018.
NICIDA, Lucia Regina de Azevedo. A medicalização do parto no Brasil a partir do estudo de manuais de obstetrícia. História, Ciências, Saúde Manguinhos, Rio de Janeiro, v.25, n.4, out.-dez. 2018, p.1147-1154.

\section{Resumo}

Nesta nota de pesquisa apresento um relato preliminar da pesquisa de doutorado em saúde da criança e da mulher que estou realizando no Instituto Nacional de Saúde da Mulher, da Criança e do Adolescente Fernandes Figueira/Fiocruz. Elegi como objeto de estudo a difusão dos saberes e práticas relativas ao parto. Optei em realizá-lo por meio da análise dos manuais de obstetrícia. Analiso manuais publicados no Brasil, entre 1980 e 2011, e pretendo contribuir para a construção de uma assistência ao parto no qual a mulher e suas necessidades sejam o centro no processo de tomada de decisões sobre os cuidados, intervenções e procedimentos a serem dispensados no trabalho de parto e no parto.

Palavras-chave: medicalização; assistência ao parto; saúde da mulher e da criança.

\begin{abstract}
In this research note, I present a preliminary account of doctoral research in women's and children's health at the Instituto Nacional de Saúde da Mulher, da Criança e do Adolescente Fernandes Figueira/Fiocruz. The aim is to study the spread of knowledge and practices relating to childbirth by analyzing obstetrics handbooks published in Brazil between 1980 and 2011, contributing to the development of childbirth care in which the woman and her needs are at the center of the decision-making process about which care, interventions, and procedures are to be dispensed during labor and childbirth.
\end{abstract}

Keywords: medicalization; childbirth care; women's and children's health. 


\section{Refletindo sobre a medicalização do parto}

Desde a década de 1980, a reflexão sobre o processo de medicalização da assistência ao parto tem se tornado recorrente na pauta de discussão, tanto nas áreas de biomédica, antropologia e política, como no âmbito dos movimentos sociais, revelando diferentes conflitos e posicionamentos. Compondo essa movimentação, destaco a realização de três conferências internacionais de consenso organizadas pela Organização Pan-Americana de Saúde (Opas) e os Escritórios Regionais da Europa e Américas da Organização Mundial da Saúde (OMS) sobre o uso apropriado de tecnologia no pré-natal, no parto e no nascimento (Boareto, 2003). A segunda delas, realizada em 1985, em Fortaleza, reconheceu o parto como um evento natural e normal, afirmando que não havia justificativas para taxas de cesárea acima de 10\% a 15\% (WHO, 1985). Além do incentivo ao parto vaginal, recomendou mudanças nas rotinas marcadas por intervenções técnicas e tecnológicas, alertando que, quando utilizadas de forma desnecessária e excessiva, poderiam causar danos tanto para a mãe quanto para o bebê (Diniz, 2005; Maia, 2010). No Brasil, a crítica às consequências perniciosas desse processo se constituiu, desde então, como um movimento que foi denominado de "humanização do parto", tendo como uma de suas características a complexidade, já que passou a envolver uma multiplicidade de questões e agentes.

Integrando essa movimentação, ganharam força os estudos científicos de avaliação das práticas de assistência ao parto, tais como a colaboração internacional baseada na metodologia de revisão sistemática, em um movimento por uma medicina baseada em evidências científicas (MBE). Em 1989, como um dos resultados dessa colaboração, foi publicada uma revisão sistemática de cerca de 40 mil estudos envolvendo 275 práticas de assistência perinatal, em um trabalho que incluiu mais de quatrocentos pesquisadores entre obstetras, pediatras, enfermeiros, estatísticos, epidemiologistas, cientistas sociais e parteiras. A pesquisa foi desenvolvida com estudos publicados desde 1950 sobre o tema gravidez e parto (Boareto, 2003; Vasconcelos, 2010).

Em 1996, a OMS publica uma coletânea dessas revisões, passando a ser reconhecidas como "Recomendações da OMS" (Diniz, 2005). As recomendações serviram de referência para a elaboração das políticas de parto brasileiras, para as campanhas do Ministério da Saúde (MS) e para os movimentos sociais pela "humanização", que se tornaram cada vez mais atuantes. Ainda em 1996, elas foram publicadas pelo MS com o título "Assistência ao parto normal: um guia prático", e no ano 2000 foi distribuída para os ginecologistasobstetras e enfermeiras obstetrizes do país (Diniz, 2005; Deslandes, 2005). Outrossim, também surgiram discussões sobre a necessidade de implementação de melhorias na interação entre a parturiente e os profissionais envolvidos nos cuidados (Diniz, 2005; Torquinst, 2002).

Contudo, a despeito dos movimentos e das transformações ocorridas nos cuidados dispensados no campo da saúde materna e neonatal e das recomendações que vêm sendo preconizadas de boas práticas de atenção obstétrica, a assistência ao parto na rede pública continua vivendo percalços: ainda é marcada pela insegurança; por uma relação profissionalusuária autoritária, com formas de tratamento discriminatório; pela banalização da dor e do sofrimento, reforçando a associação do parto vaginal a uma experiência traumática. Por 
outro lado, no setor privado, o que se destaca é o alto percentual de cesarianas realizadas, chegando em 2015 a cerca de 85\% (Rehuna, 2015).

Para analisar o quadro assistencial brasileiro, foi realizada, entre 2011 e 2012, a pesquisa "Nascer no Brasil: Inquérito Nacional sobre Parto e Nascimento", sob a coordenação da Fundação Oswaldo Cruz. Entre os resultados relativos às intervenções obstétricas no trabalho de parto e no parto, a pesquisa apontou, por exemplo, um uso de episiotomia de 56,1\% entre as mulheres que participaram do estudo, número que sobe para quase $75 \%$ quando consideradas as primíparas. Taxas distantes do que vem sendo recomendado pela OMS, que seria em torno de 10\% e 30\% (Leal et al., 2014).

Outra intervenção que apresentou elevada prevalência foi a utilização da posição de litotomia durante o parto, atingindo 90\% quando consideradas as parturientes de risco habitual, enquanto estudos indicam que as posições verticalizadas trazem maior benefício para a mulher e para o feto (Leal et al., 2014). Dados como esses nos mostram um longo caminho a ser percorrido para que a mulher desfrute de uma assistência que atenda suas necessidades.

A partir desse quadro, considero que um estudo sócio-histórico da medicalização do parto, relacionado à pesquisa documental, reconhecendo a riqueza de informações que ela pode nos fornecer (Cellard, 2012), em muito tem a contribuir para compreender o cenário obstétrico brasileiro atual. Assim, partindo da hipótese de que a compreensão do modelo medicalizado de assistência ao parto passa pelo entendimento e pela identificação dos paradigmas que têm sido difundidos pela ciência obstétrica, e considerando que os manuais de obstetrícia são um importante veículo de disseminação do embasamento teórico que serve de sustentação às práticas, estabeleci como pergunta geral de pesquisa: quais cuidados, procedimentos e indicações de realização de intervenções obstétricas aparecem descritos nos manuais de obstetrícia publicados no Brasil?

A reflexão sobre essa pergunta geral nos indica inúmeras possibilidades de caminhos de investigação; dentre elas elegi aquela que busca identificar como os manuais têm orientado quanto à realização de intervenções obstétricas referentes à assistência ao trabalho de parto e ao parto. Qual a relação entre essas orientações e as evidências científicas existentes em cada período? Como tem se delineado a incorporação e exclusão das intervenções obstétricas identificadas? Quais atores compõem a cena do parto e qual função desempenham no processo de parturição?

\section{Sobre os estudos dos manuais}

Alguns estudos sugerem que os manuais funcionariam como instrumentos de transmissão de conhecimento científico sistematizado, apresentando o corpo teórico aceito pelo campo, assim como as suas aplicações. Dessa forma, esses manuais podem funcionar como mecanismo de conservação de bases teóricas dominantes, definindo o que se constitui como problema e como solução em determinada área do saber (Kuhn, 2011).

Nesse sentido, julgo importante investigar quais teorias e aplicações estão sendo difundidas nos manuais de obstetrícia publicados entre os anos 1980 e 2011 e se estão relacionados, ou não, ao modelo de assistência que se tornou hegemônico na segunda 
metade do século XX, e que ainda faz parte de nossa realidade, modelo no qual intervenções técnicas e tecnológicas são frequentemente utilizadas de forma desnecessária e excessiva (Diniz, 2005; Maia, 2010). Quanto ao recorte cronológico, estabeleci como ponto de partida a década de 1980, por ser um período em que ocorre uma profusão de movimentos de reflexão sobre o processo de medicalização do parto, como as conferências de consenso apoiadas pela OMS, e como final o ano de 2011, por ser o ano de realização da pesquisa "Nascer no Brasil", que apresenta um retrato da situação da atenção ao parto e nascimento.

Minha proposta segue a linha dos estudos realizada por Nakano (2015) ao proceder às análises do capítulo "Operação cesariana", em três edições da publicação de Jorge de Rezende intitulada Obstetrícia; do trabalho de Martin (2006) que analisa as diferentes descrições sobre o trabalho de parto, e suas transformações no decorrer das diferentes edições do Williams obstetrics; e do trabalho de Arredondo González (2015) que investiga oito manuais publicados em língua castelhana entre os anos de 1855 e 1955, para identificar as tecnologias e objetos utilizados nos cuidados de enfermos e o reconhecimento social daqueles que os utilizavam.

O estudo dos manuais de obstetrícia como instrumento pedagógico permite-nos uma aproximação das concepções validadas pela ciência obstétrica que estão sendo recomendadas aos médicos em um determinado tempo e em um determinado espaço (Nakano, 2015). A partir desse conhecimento poderei identificar, analisar e sistematizar os argumentos e as estratégias que têm contribuído para o processo de medicalização da assistência ao parto no Brasil.

Iniciando a coleta de dados, realizei uma busca nos portais das bibliotecas das principais universidades de medicina do país: Universidade Federal do Rio de Janeiro, Universidade de São Paulo, Universidade Estadual de Campinas; assim como na biblioteca da Fundação Oswaldo Cruz, onde localizei 56 títulos disponíveis para consulta e cuja lista provisória está especificada na Tabela 1.

Tabela 1: Lista provisória de títulos de manuais localizados

\begin{tabular}{lll}
\hline No $^{\circ}$ & Título & Autor/Editor/Organizador \\
\hline 1 & Assistência ao parto e tocurgia: manual de orientação & Camano, L. et al. \\
\hline 2 & Assistência obstétrica: um guia prático & Fraser, D.M. et al. \\
\hline 3 & Compêndio de obstetrícia e ginecologia & Norwitz, E.R. et al. \\
\hline 4 & Compêndio Ill: atualização em obstetrícia e ginecologia & American College of Obstetricions and Gynecologists \\
\hline 5 & Condutas em clínica obstétrica & Madi, J.M. et al. \\
\hline 6 & Condutas em obstetrícia & Rolnik, D.L. et al. \\
\hline 7 & Condutas em obstetrícia & Viggiano, M.G.C. \\
\hline 8 & Diagnóstico e tratamento em obstetrícia e ginecologia & Benson, R.C. \\
\hline 9 & Fundamentos de ginecologia e obstetrícia & Hacker, N.F.; Moore, J.G. \\
\hline 10 & Ginecologia e obstetrícia de Netter & Smith, R.P. \\
\hline 11 & Ginecologia: manual de normas e condutas & Lopes, R.G.C. et al. \\
\hline
\end{tabular}


Tabela 1: Lista provisória de títulos de manuais localizados (cont.)

\begin{tabular}{|c|c|c|}
\hline No & Título & Autor/Editor/Organizador \\
\hline 12 & Manual de assistência ao parto & Cabral, A.C.V. et al. \\
\hline 13 & Manual de condutas em obstetrícia & Chaves Netto, H.; Sá, R.A.M. \\
\hline 14 & Manual de ginecologia e obstetrícia & Ostergard, D., Brangonier, J.R. \\
\hline 15 & Manual de ginecologia e obstetrícia do Johns Hopkins & Lambrou, N.C. et al. \\
\hline 16 & Manual de obstetrícia & Basegio, D.L. et al. \\
\hline 17 & Manual de obstetrícia & Ellis, J.W.; Beckmann, C.R.B. \\
\hline 18 & Manual de obstetrícia & Evans, A.T. \\
\hline 19 & Manual de obstetrícia & Martins, J.A.P. \\
\hline 20 & Manual de obstetrícia de Williams & Leveno, K.J. et al. \\
\hline 21 & Manual de obstetrícia diagnóstico e conduta & Mathias, L. \\
\hline 22 & Manual de obstetrícia e ginecologia & Benson, R.C. \\
\hline 23 & Manual de obstetrícia: diagnóstico e tratamento & Niswander, K.R.; Evans, A.T. \\
\hline 24 & Manual de terapêutica: ginecologia e obstetrícia & Grando, L.B. \\
\hline 25 & Manual Febrasgo - 2000 & $\begin{array}{l}\text { Federação Brasileira das Sociedades de Ginecologia } \\
\text { e Obstetrícia }\end{array}$ \\
\hline 26 & Manual prático de monitoração obstétrica & Santos, P.R.F. \\
\hline 27 & Noções práticas de obstetrícia & Corrêa, M.D. \\
\hline 28 & Obstetrícia & Barros, D.N. et al. \\
\hline 29 & Obstetrícia & Cabral, A.C.V. \\
\hline 30 & Obstetrícia & Falcone, I. \\
\hline 31 & Obstetrícia & Lemoine, J.P. et al. \\
\hline 32 & Obstetrícia & Moron, A.F. et al. \\
\hline 33 & Obstetrícia & Rezende, J. \\
\hline 34 & Obstetrícia & Rezende Filho, J.; Montenegro, C.A.B. \\
\hline 35 & Obstetrícia \& ginecologia - Manual para o residente & Mariani Neto, C.; Tadini, V. \\
\hline 36 & $\begin{array}{l}\text { Obstetrícia: fundamentos e avanços na propedêutica, } \\
\text { diagnóstico e tratamento }\end{array}$ & Aldrighi, J.M. et al. \\
\hline 37 & Obstetrícia básica & Neme, B. \\
\hline 38 & Obstetrícia básica & Chaves Netto, H.; Sá, R.A.M. \\
\hline 39 & Obstetrícia diagnóstico e tratamento & Santos, L.C. et al. \\
\hline 40 & Obstetrícia e ginecologia: diagnóstico e tratamento & DeCherney, A.H.; Nathan, L. \\
\hline 41 & Obstetrícia fundamental & Rezende, J.; Montenegro, C.A.B. \\
\hline 42 & Obstetrícia fundamental & Rezende Filho, J.; Montenegro, C.A.B. \\
\hline 43 & Obstetrícia normal & Briquet, $\mathrm{R}$. \\
\hline 44 & Obstetrícia normal Briquet & Briquet, R. et al. \\
\hline 45 & Obstetrícia normal Briquet & Guariento, A.; Delascio, D. \\
\hline
\end{tabular}


Tabela 1: Lista provisória de títulos de manuais localizados (cont.)

\begin{tabular}{lll}
\hline No $^{\circ}$ & Título & Autor/Editor/Organizador \\
\hline 46 & Obstetrícia normal e patológica & Barbosa, L.A.H. \\
\hline 47 & Obstetrícia protocolos de diagnóstico e tratamento & Mendonça, D. \\
\hline 48 & Obstetrícia gestações normais \& patológicas & Gabbe, S.G. et al. \\
\hline 49 & Obstetrícia: texto básico para estudantes de medicina & Grelle, F.C. et al. \\
\hline 50 & $\begin{array}{l}\text { Protocolos assistenciais Clínica Obstétrica da Faculdade } \\
\text { de Medicina da USP }\end{array}$ & Zugaib, M.; Bittar, R.E. \\
\hline 51 & Revisão em ginecologia e obstetrícia do Johns Hopkins & Bankowski, B.J. et al. \\
\hline 52 & Rotinas em obstetrícia & Freitas, F. et al. \\
\hline 53 & Rotinas em obstetrícia & Montenegro, C.A.B. \\
\hline 54 & Tratado de obstetrícia Febrasgo & $\begin{array}{l}\text { Federação Brasileira das Sociedades de Ginecologia } \\
\text { e Obstetrícia }\end{array}$ \\
\hline 55 & Williams obstetrícia & Cunningham. F.G. et al. \\
\hline 56 & Zugaib obstetrícia & Zugaib, M. \\
\hline
\end{tabular}

Fonte: elaborada pela autora.

$\mathrm{Na}$ análise preliminar das fontes encontrei indícios de que os saberes e práticas difundidos pelos manuais de obstetrícia têm correspondência com um modelo de assistência que transforma algumas intervenções em procedimento de rotina. Sobre a realização de episiotomia, por exemplo, foram encontradas indicações de uso sem restrições, especialmente nos casos das primigestas. Esse posicionamento foi encontrado em Rezende (2002, p.340):

É a episiotomia, quase sempre, indispensável nas primiparturientes, e nas multíparas nas quais tenha sido anteriormente praticada. Feita com tesoura ou bisturi, poderá ser mediana (perineotomia), médio-lateral, que tem nossas preferências, e lateral (Fig. 6); a ferida incisa, de mais fácil e segura recomposição, substitui-se a lesão contusa da rotura.

E, mais recentemente, em Kondo (2011, p.635):

Nas primigestas, preconiza-se fórcipe baixo ou de alívio com episiotomia médio-lateral (direita ou esquerda) ou perineotomia. A episitomia deve ser realizada quando a cabeça fetal força e entreabre a fenda vulvar, sem distendê-la excessivamente. A extensão da episiotomia deve ser suficiente para permitir o despreendimento fetal e evitar o trauma materno. Nas multíparas, a episiotomia é seletiva, podendo ou não ser realizada, de acordo com a elasticidade do períneo e o tamanho fetal.

As orientações sobre a posição no momento do parto seguem o mesmo modelo. Nesse caso, a indicação de uso da posição litotômica continua sendo recomendada como a "mais satisfatória" e "preferencial", como pode ser visto no trecho:

O parto pode ser realizado com a mãe em várias posições. A mais usada e frequentemente a mais satisfatória é a posição de litotomia dorsal a fim de aumentar o diâmetro da saída pélvica (Cap. 3). Em muitas salas de parto isto é feito com a mulher deitada no leito. Para melhor exposição são usados porta-pernas ou estribos (Cunningham et al., 2000, p.286). 
Assim como em Kondo (2011, p.634), em que encontramos: “A posição preferencial é a de litotomia, com apoio da panturrilha na perneira. Na cama-mesa de LDR, a posição semissentada com apoio de pé também é utilizada. ... O obstetra deve, preferencialmente, permanecer sentado para realizar o parto".

Na análise de tais recomendações não foi encontrada menção a procedimentos que nos remetessem a uma atuação ativa da mulher em relação às decisões e procedimentos adotados. Tampouco nenhum canal ou rotina que indicasse alguma possibilidade de expressão da mulher sobre suas necessidades e/ou desejos.

Nesse sentido, a própria definição do que seria a função da ciência obstétrica, encontrada em alguns manuais, poderia estar contribuindo para tal configuração. Como pode ser visto em Rezende, em que o autor destaca:

Obstetrícia é a parte da Medicina que estuda os fenômenos da reprodução, na mulher. Ocupa-se, assim, com a gestação, o parto e o puerpério - investigando-lhes a fisiologia, a patologia e os acidentes - e dita as regras de sua assistência, em circunstâncias normais e sob condições anômalas (Rezende, 2002, p.1; destaque no original).

E, para além da função do cuidado individual da gestante e seu bebê, a obstetrícia também é apresentada por alguns autores como a ciência responsável pela reprodução de uma nação, em que se relaciona com diferentes fatores que influenciarão a quantidade e a qualidade de seus cidadãos.

A obstetrícia é o ramo da medicina responsável pelo parto e pela assistência e tratamento da mãe antes e após o parto (Oxford English Dictionary, 1993). Portanto, a disciplina está relacionada principalmente com a gravidez, o trabalho de parto e o puerpério em circunstâncias normais e anormais. Em um sentido mais amplo, a obstetrícia está relacionada à reprodução de uma nação, porque está relacionada a todos os fatores fisiológicos, patológicos, psicológicos e sociais que influenciam profundamente tanto a quantidade quanto a qualidade de novas gerações de cidadãos (Cunningham et al., 2000, p.1).

Visto a obstetrícia chamar para si a função de "ditar" regras e de responsabilizar-se pela tomada de decisão dos procedimentos relativos ao parto, mesmo em "circunstâncias normais", torna-se possível inferir que isso tende a gerar situações assistenciais em que o(a) obstetra terá o poder de conduzir a assistência sem que haja consulta ou discussão com a mulher ou com quaisquer outros atores que venham a fazer parte da cena do parto.

Para finalizar, gostaria de ressaltar a importância do estudo dos manuais de obstetrícia como fonte de análise dos saberes e práticas relativos ao parto que têm sido sistematizados e validados pela ciência obstétrica, que ali estão sendo difundidos. Estudo este que nos convida ao exame e identificação dos aspectos que estão sendo recomendados aos médicos na fase de "educação científica" e que, possivelmente, têm sido incorporados como sua prática assistencial. Nesse sentido, a pesquisa proposta pode abrir novas perspectivas de pensar e agir na assistência ao parto e, em um sentido mais amplo, contribuir com as iniciativas de âmbito nacional para garantir a assistência integral à saúde da mulher e da criança, dispensando uma atenção à gravidez, ao parto e ao puerpério, em que prevaleçam as boas condutas obstétricas cujo foco esteja nas necessidades vividas e sentidas por cada uma das mulheres. 


\section{REFERÊNCIAS}

ARREDONDO GONZÁLES, Claudia Patricia. Cuidados y mundo material: El caso de la enfermería y latecnologia (1855-1955). Tese (Doctorado y la enfermería) - Facultad de Ciencias de La Salud, Alicante. 2015.

BOARETO, Maria Cristina. Avaliação da política de humanização ao parto $e$ nascimento no município do Rio de Janeiro. Rio de Janeiro. Dissertação (Mestrado em Saúde Pública) - Escola Nacional de Saúde Pública, Fundação Oswaldo Cruz, Rio de Janeiro. 2003.

CELLARD, André.

A análise documental. In: Poupart, Jean et al. $A$ pesquisa qualitativa: enfoques epistemológicos e metodológicos. Petrópolis: Vozes. p.295-315. 2012.

CUNNINGHAM, F. Gary et al.

Williams obstetrícia. Rio de Janeiro: Guanabara Koogan. 2000.

DESLANDES, Suely Ferreira.

A ótica de gestores sobre a humanização da assistência nas maternidades municipais do Rio de Janeiro. Ciência \& Saúde Coletiva, v.10, n.3, p.615-626. 2005.

DINIZ, Simone Grilo.

Humanização da assistência ao parto no Brasil: os muitos sentidos de um movimento. Ciência \& Saúde Coletiva, v.10, n.3, p.627-637. 2005.

KONDO, Mario Macoto.

Assistência ao parto. In: Zugaib, Marcelo. Protocolos assistenciais, clinica obstétrica, FMUSP. São Paulo: Atheneu. p.631-636. 2011.

KUHN, Thomas S.

A estrutura das revoluções científicas. São Paulo: Perspectiva. 2011.

LEAL, Maria do Carmo et al.

Intervenções obstétricas durante o trabalho de parto e parto em mulheres brasileiras de risco habitual. Caderno de Saúde Pública, v.30, supl.1, p.S17-S32. Disponível em: <http://www.scielo. br/pdf/csp/v30s1/0102-311X-csp-30-s1-0017. pdf $>$. Acesso em: 10 out. 2015. 2014.
MAIA, Mônica Bara.

Humanização do parto: política pública, comportamento organizacional e ethos profissional. Rio de Janeiro: Editora Fiocruz. p.19-49. Disponível em: <http://static.scielo.org/ scielobooks/pr84k/pdf/maia-9788575413289. pdf $>$. Acesso em: 21 maio 2016. 2010.

MARTIN, Emily.

A mulher no corpo: uma análise cultural da reprodução. Rio de Janeiro: Garamond. 2006.

NAKANO, Andreza Rodrigues.

Uso de tecnologias e as transformações nas práticas sociais e de nascimento no Brasil: uma normalização da cesárea? Tese (Doutorado em Ciências) - Instituto Nacional de Saúde da Mulher, da Criança e do Adolescente Fernandes Figueira, Rio de Janeiro. 2015.

REHUNA.

Rede pela Humanização do Parto e Nascimento. Convite para a IV Conferência Internacional de Humanização do Parto e Nascimento de 2016. Disponível em: <http://www.rehuna.org.br/ index.php/seminario/eventos/57-iv-conferenciainternacional-de-humanizacao-do-parto-enascimento >. Acesso em: 2 out. 2015. 2015.

REZENDE, Jorge de.

Obstetrícia. Rio de Janeiro: Editora Guanabara Koogan. 2002.

TORQUINST, Carmen Susana.

Armadilhas da nova era: natureza e maternidade no ideário da humanização do parto. Revista de Estudos Feministas, v.10, n.2, p.483-492. 2002.

VASCONCELOS, Alessandra Alves de. A atuação das enfermeiras na humanização do parto e nascimento no Distrito Federal. Brasília. Dissertação (Mestrado em Ciências da Saúde) Universidade de Brasília, Brasília. 2010.

WHO.

World Health Organization. Appropriate technology for birth. Lancet, v.24, n2, p.436-437. 1985.

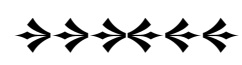

